\title{
A comparison of perceptually motivated loss functions for binary mask estimation in speech separation
}

\author{
Danny Websdale, Ben Milner \\ University of East Anglia \\ d.websdale@uea.ac.uk, b.milnerduea.ac.uk
}

\begin{abstract}
This work proposes and compares perceptually motivated loss functions for deep learning based binary mask estimation for speech separation. Previous loss functions have focused on maximising classification accuracy of mask estimation but we now propose loss functions that aim to maximise the hit minus false-alarm (HIT-FA) rate which is known to correlate more closely to speech intelligibility. The baseline loss function is binary cross-entropy (CE), a standard loss function used in binary mask estimation, which maximises classification accuracy. We propose first a loss function that maximises the HIT-FA rate instead of classification accuracy. We then propose a second loss function that is a hybrid between CE and HIT-FA, providing a balance between classification accuracy and HIT-FA rate. Evaluations of the perceptually motivated loss functions with the GRID database show improvements to HIT-FA rate and ESTOI across babble and factory noises. Further tests then explore application of the perceptually motivated loss functions to a larger vocabulary dataset.
\end{abstract}

Index Terms: HIT-FA, speech separation, binary mask

\section{Introduction}

Speech separation from a monaural source aims to separate target speech from interfering background noise to produce a more intelligible signal. Such systems have widespread application in areas such as speech enhancement, robust speech recognition and hearing aid design $[1,2,3]$. There are two main approaches to this problem. The first is to derive a statistical model that makes certain assumptions about the background noise, and includes methods such as spectral subtraction, Weiner filtering and mean-square error estimation [4]. These approaches have been shown to not provide an increase in intelligibility for human listeners $[5,6]$. This is because distortions (e.g. musical noise) are introduced and low-intensity sounds (e.g. unvoiced consonants), which are important for intelligibility, are lost. The second approach uses computational auditory scene analysis (CASA) [7], inspired by perceptual principles of auditory scene analysis (ASA), can be effective in both stationary and non-stationary noise [8].

In CASA, speech can be extracted by applying a mask to a time-frequency (T-F) representation of noisy speech. An ideal binary mask (IBM) retains speech dominant T-F units and suppresses noise dominant T-F units. An IBM can be constructed from premixed speech and noise and defined as

$$
\operatorname{IBM}(t, f)= \begin{cases}1, & \text { if } \operatorname{SNR}(t, f) \geq \mathrm{LC} \\ 0, & \text { otherwise }\end{cases}
$$

where $t$ and $f$ represent time frame and frequency bin respectively and LC is a local criterion. T-F units dominated by speech are assumed to have a signal-to-noise ratio (SNR) greater than

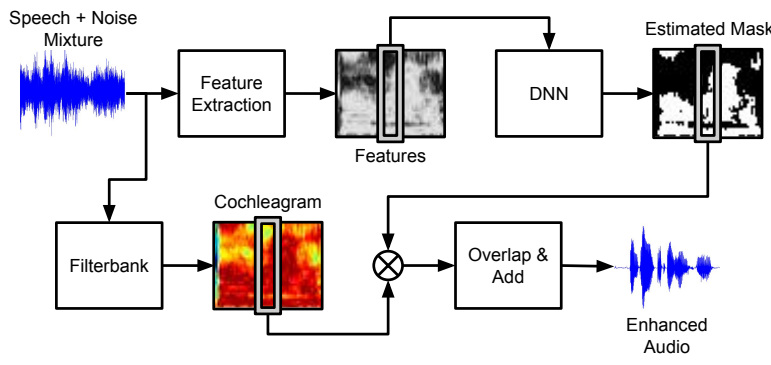

Figure 1: Overview of the speech separation system.

or equal to LC are represented by 1 and retained. Noise dominant units are assumed to be less than LC are represented by 0 and suppressed. Several studies have reported subjective test results where IBMs improve intelligibility for speech in noise for both normal-hearing and hearing-impaired listeners $[9,10,11,12]$. In practice an IBM is not available and instead the binary mask must be estimated from the noisy signal. This allows speech separation to be treated as a mask estimation problem that uses supervised learning to map acoustic features extracted from noisy speech to a binary mask [13].

Previous studies have focused on either developing improved feature extraction methods or using more sophisticated classifiers - for example moving from Gaussian mixture models (GMMs) to deep neural networks (DNNs). Some attention has been focussed on improving the classifier to reduce perceptual error by changing the loss function for text-to-speech applications [14], and introducing signal approximation loss functions $[15,16]$ as a replacement for mask approximation within speech separation applications. Signal approximation loss functions apply the output of the network to the noisy spectrum within the loss function, and minimise this with respect to the target. Signal approximation works well when the network target is the power spectrum, outperforming mask approximation, however is not applicable to a cochleagram framework, due to the cochleagram is constructed from overlapping gammatone filterbanks. In this work we focus on mask approximation loss functions within a cochleagram framework for consideration to cochlear implants.

We first compare the performance of two different feature extraction methods, namely the multi-resolution cochleagram feature (MRCG) and the complementary feature set (ARpMG) $[17,18]$. We also propose two perceptually motivated mask approximation loss functions that are inspired by the hit minus false-alarm (HIT-FA) rate, which has been shown to correlate more closely to speech intelligibility than classification accuracy $[19,20,17,18,21]$. Classification accuracy is the basis of the binary cross-entropy (CE) loss function used as standard for binary mask estimation forming our baseline.

Figure 1 shows the overall speech separation system. Features are extracted from noisy speech and input into a DNN to 
estimate a binary mask. Masking is applied to a cochleagram [7] of the noisy speech which suppresses noise-dominated T-F units and the remaining signals are overlapped and summed to produce the enhanced signal.

The remainder of the paper is organised as follows. The classifier and proposed loss functions are described in Section 2. Section 3 provides an overview of two feature extraction methods. Performance evaluations are made in Section 4 which first compare the feature extraction methods and then the effectiveness of the proposed perceptual loss functions under varying noise and SNR conditions using both a small dataset (GRID) and large dataset (RM-3000).

\section{Perceptually motivated loss functions}

The purpose of the classifier is to learn a mapping between acoustic features extracted from the noisy speech mixture and the binary mask output. Previous studies have shown a progression in classifiers used, beginning with GMMs through to support vector machines (SVMs), multilayer perceptrons (MLPs) and finally deep learning $[19,20,17,18,21,22]$. We use DNNs as the classifier in this work which normally uses the binary cross-entropy (CE) loss function in training for classification tasks. The DNN uses rectified linear units for hidden layers and a sigmoid layer for the output. The CE loss function is now reviewed and two new perceptually motivated loss functions introduced inspired by the HIT-FA rate.

\subsection{Binary cross-entropy $(\mathrm{CE})$}

Binary cross-entropy (CE) is a standard loss function used within DNN training for classification tasks [23] and forms the baseline loss function. The aim of CE is to maximise the accuracy of the estimated mask where accuracy is defined as the proportion of correctly labeled T-F units. The CE loss, $\mathrm{L}^{\mathrm{CE}}$, is calculated as

$$
\mathrm{L}^{\mathrm{CE}}=-\frac{1}{N} \sum_{n=1}^{N}\left[y_{n} \log \left(\hat{y}_{n}\right)+\left(1-y_{n}\right) \log \left(1-\hat{y}_{n}\right)\right]
$$

where $\boldsymbol{y}$ and $\hat{\boldsymbol{y}}$ are vectors that comprise concatenated frames of T-F units for each mini-batch in DNN training, from the IBM and estimated mask respectively. Each of these vectors comprises $N$ T-F units which are indexed by $n$.

\subsection{HIT-FA (HF)}

Our first perceptually motivated loss function (HF) is based on maximising the HIT-FA rate, which several studies have shown correlates more closely to intelligibility than mask accuracy $[19,20,17,18,21]$. In terms of the loss function, HITs refer to the proportion of correctly labeled target-dominant T-F units while FAs refer to the proportion of incorrectly labeled noisedominant T-F units. Studies have shown that achieving high HITs and low FAs produces higher intelligibility [19].

The key difference between the CE and HF loss functions is that $\mathrm{CE}$ calculates accuracy over all T-F units together, whereas HF calculates the accuracy of target-dominant (1) and noisedominant (0) T-F units separately. HIT-FA has a range between 1 and -1 , with 1 being best performance. However within DNN training loss is minimised, therefore we use FA-HIT to give best performance at -1 and remove this discrepancy. The HIT-FA loss, $\mathrm{L}^{\mathrm{HF}}$, is calculated as

$$
\mathrm{L}^{\mathrm{HF}}=\frac{1}{S} \sum_{n=1}^{N}\left[\left(1-y_{n}\right) \hat{y}_{n}\right]-\frac{1}{R} \sum_{n=1}^{N}\left[y_{n} \hat{y}_{n}\right]
$$

where $S$ is the number of T-F units within $y$ that should be suppressed (0s) and $R$ is the number of T-F units within $\boldsymbol{y}$ that should be retained (1s).

\subsection{Binary cross-entropy HIT-FA hybrid (CHF)}

Within an IBM the number of retained T-F units, $R$, and number of suppressed units, $S$, are generally not equal. In most cases there are more noise-dominant T-F units than target-dominant units, due mainly to areas of non-speech. The HF loss function is calculated as proportions of $R$ and $S$ separately, and is therefore less affected by bias towards a difference between $R$ and $S$. Conversely, the CE loss function is calculated as an overall accuracy of $R$ and $S$ and is therefore biased towards the greater of the two. We take inspiration from the HF loss function to produce a hybrid cross-entropy HIT-FA (CHF) loss function by modifying the CE function to remove this bias. To do this we normalise the ratio between $R$ and $S$ such that $R=S$. This is achieved by multiplying the portion related to $S$ by $R / S$. The cross-entropy HIT-FA loss function, $\mathrm{L}^{\mathrm{CHF}}$, is calculated as

$$
\mathrm{L}^{\mathrm{CHF}}=-\frac{1}{N} \sum_{n=1}^{N}\left[y_{n} \log \left(\hat{y}_{n}\right)+\frac{R}{S}\left(1-y_{n}\right) \log \left(1-\hat{y}_{n}\right)\right]
$$

Our data has a bias towards $S$, therefore this normalisation will cause an increase of HITs at a cost of increasing FAs. The opposite would occur if the bias was towards $R$. A reduction to overall classification accuracy will occur in all cases where $R \neq S$ prior to normalisation.

\section{Feature extraction}

Feature extraction aims to identify suitably discriminative information in the noisy input speech that enables the DNN to determine whether T-F units are target (1) or noise (0) dominated. We investigate two different acoustic features.

\subsection{Multi-resolution cochleagram feature (MRCG)}

The MRCG feature was designed specifically for mask estimation and combines four cochleagrams at different resolutions [17]. The first captures high resolution localised detail while the remaining cochleagrams capture lower resolution spectrotemporal content. Cochleagrams are computed by passing the input signal through a 64-channel gammatone filterbank [24].

The outputs from the gammatone filterbank are split into $20 \mathrm{~ms}$ frames with $10 \mathrm{~ms}$ frame shift with power spectrum computed followed by a log which gives the first cochleagram, $C G_{1}$. Similarly, $C G_{2}$ uses $200 \mathrm{~ms}$ frames with $10 \mathrm{~ms}$ frame shift. Finally, $C G_{3}$ and $C G_{4}$ are derived by applying an $11 \times 11$ and $23 \times 23$ mean filter kernel to $C G_{1}$ [17]. The final MRCG feature, $\boldsymbol{x}^{\mathrm{MRCG}}$, is produced by stacking all four $C G \mathrm{~s}$.

\subsection{Complementary feature set (ARpMG)}

The complementary feature set (ARpMG) is an ensemble of commonly used acoustic features [17, 18, 21]. This combines amplitude modulation spectrum (AMS) [25, 26, 19], relative spectral transformed perceptual linear prediction (RASTAPLP) [27] and mel-frequency cepstral coefficients (MFCCs) [28] with a gammatone filterbank (GFB) [17].

The specific implementation is taken from [18] where AMS features are computed from $32 \mathrm{~ms}$ frames with $10 \mathrm{~ms}$ frame shift to give a 15-D vector. RASTA-PLP features are also computed from $32 \mathrm{~ms}$ frames with $10 \mathrm{~ms}$ frame shift and result in 
a 13-D vector. MFCCs are computed from $20 \mathrm{~ms}$ frames with $10 \mathrm{~ms}$ frame shift with a 30-D vector produced.The GFB feature is computed from a 64-channel gammatone filterbank, decimating to $100 \mathrm{~Hz}$ to give a $10 \mathrm{~ms}$ frame shift and results in a $64-\mathrm{D}$ vector. Combining these gives the 122-D ARpMG feature, $\boldsymbol{x}^{\mathrm{ARpMG}}$, which is produced at a $10 \mathrm{~ms}$ frame rate.

\subsection{Temporal information}

Including temporal information with static features have shown to improve performance for automatic speech recognition (ASR) $[29,30]$. In this work we include temporal information via vector stacking. Given a sequence of static feature vectors, $\left\{\ldots, \boldsymbol{x}_{i-2}, \boldsymbol{x}_{i-1}, \boldsymbol{x}_{i}, \boldsymbol{x}_{i+1}, \boldsymbol{x}_{i+2}, \ldots\right\}$, neighbouring vectors within a window that extends $K$ vectors either side of the current vector are stacked, i.e.

$$
\boldsymbol{x}_{i}^{\mathrm{STACK}}=\left[\boldsymbol{x}_{i-K}, \ldots, \boldsymbol{x}_{i}, \ldots, \boldsymbol{x}_{i+K}\right]
$$

Preliminary tests found a window of 7 frames (i.e. $K=3$ ) gave best performance.

\section{Experimental results}

Experiments first identify the best performing feature set, then compare the performance of the proposed loss functions within a small dataset (GRID) across a range of noises and SNRs. This is then expanded to a larger vocabulary dataset (RM-3000).

Initial tests use the GRID database which contains recordings from 34 speakers who each produced 1000 sentences [31]. Each sentence comprises six words and follows the grammar shown in Table 1. Speaker 12 (male) was selected for the evaluations and the audio downsampled to $16 \mathrm{kHz}$. The speech database is split into 200 test sentences, and 800 training sentences of which 160 are removed for validation within training.

Table 1: GRID sentence grammar.

\begin{tabular}{|c|c|c|c|c|c|}
\hline command & colour & preposition & letter & digit & adverb \\
\hline \hline bin & blue & at & A-Z & $1-9$ & again \\
lay & green & by & minus W & zero & now \\
place & red & in & & & please \\
set & white & with & & & soon \\
\hline
\end{tabular}

The second dataset, RM-3000 [32], consists of 3000 sentences spoken by a single native English speaking male speaker. The sentences were randomly selected from the 8000 sentences in the Resource Management (RM) Corpus [33]. The vocabulary size of 1000 words and no strict grammar give a more realistic environment, and more challenging task when compared to GRID. The audio was downsampled to $16 \mathrm{kHz}$ and the speech database is split into 600 test sentences, and 2400 training sentences of which 480 are removed for validation within training.

A range of DNN parameters were explored to find the best performing network and are shown in Table 2. From these, 30 different network configurations were randomly selected and the best performing configuration based on the validation set chosen. This procedure was repeated for all loss functions with babble noise at $-5 \mathrm{~dB}$, so that the DNN configuration for each loss function can be considered optimised. The optimal parameters for each loss function was used in subsequent experiments for the remaining noise types and SNRs.

For evaluating the performance of our speech separation systems, we utilise three objective measures: i) classification accuracy, ii) HIT-FA rate and iii) ESTOI [34]. Within the
ESTOI function, non-speech frames are removed via dynamic range thresholding, however in our experiments, we found this method to perform poorly and not remove the desired nonspeech frames. Therefore, we remove the non-speech frames using the alignment transcriptions provided for each dataset prior to the ESTOI function.

Table 2: Parameter set for DNN optimisation
\begin{tabular}{|c|c|}
\hline Parameter & value \\
\hline \hline hidden layers & $3,4,5$ \\
hidden units & 1024 \\
mini-batch size & $256,512,1024$ \\
hidden dropout & $0.0,0.2,0.5$ \\
learning rate & $3 e^{-4}, 1 e^{-4}, 3 e^{-5}, 1 e^{-5}$ \\
momentum & $0.0,0.9,0.99$ \\
\hline
\end{tabular}

\subsection{Analysis of features}

These tests compare the performance of our two feature extraction methods on the GRID dataset. Experiments are performed in babble noise at an SNR of $-5 \mathrm{~dB}$ with LC set to $-10 \mathrm{~dB}$. An LC $5 \mathrm{~dB}$ lower than the overall SNR was found to give best performance in a initial tests and conforms to that described in $[18,21]$. Table 3 shows the objective performance for the two feature sets with all loss functions.

Table 3: Classification accuracy (in \%), HIT-FA (in \%) and ESTOI scores for the feature comparison using the GRID dataset in babble noise at $-5 \mathrm{~dB}$.

\begin{tabular}{|c|c|c|cr|c|}
\hline Feature & Loss & Acc & \multicolumn{2}{|c|}{ HIT-FA (FA) } & ESTOI \\
\hline \hline \multirow{3}{*}{ MRCG } & CE & $\mathbf{8 9 . 7}$ & 66.7 & $(4.6)$ & $\mathbf{4 6 . 9}$ \\
& HF & 84.8 & 68.0 & $(14.5)$ & 42.6 \\
& CHF & 88.3 & $\mathbf{7 1 . 7}$ & $(9.5)$ & 46.1 \\
\hline \multirow{3}{*}{ ARpMG } & CE & $\mathbf{8 7 . 9}$ & 60.6 & $(5.4)$ & $\mathbf{4 1 . 6}$ \\
& HF & 83.1 & $62.2(15.1)$ & 37.7 \\
& CHF & 85.5 & $\mathbf{6 5 . 3}(12.0)$ & 39.8 \\
\hline \multicolumn{5}{|c|}{ unprocessed audio } & 20.3 \\
\hline
\end{tabular}

Performance shows that across all loss functions and objective measures, the MRCG feature extraction method outperforms the ARpMG method. Intelligibility gains can be found for all features over that of unprocessed.

\subsection{Analysis of perceptual loss functions}

These tests compare the performance of our proposed perceptual loss functions using the MRCG feature (see Section 4.1). Experiments are performed in babble and factory noise at SNRs of $-5 \mathrm{~dB}, 0 \mathrm{~dB}$ and $+5 \mathrm{~dB}$, with LC set to $5 \mathrm{~dB}$ lower than the selected SNR with the GRID dataset. Table 4 shows the objective performance across all experiments.

Focusing first on classification accuracy, the CE loss function gives highest accuracy across all SNRs and noise types. This is expected as the CE loss function is targeted to maximise accuracy. The hybrid CHF loss function has accuracy almost as high as CE and exceeds that of HF which is not designed to maximise classification accuracy.

Considering now the HIT-FA rate, the HF loss function now outperforms the $\mathrm{CE}$ loss function as it is designed to maximise HIT-FAs. However, the hybrid CHF loss function gives even 
higher HIT-FAs across all SNRs and noise types. In terms of HITs, the CHF and HF loss functions perform similarly, but their main difference is that the CHF loss function generates less FAs compared to the HF loss function. Lowest HITs and FAs are found with the CE loss function due to it favouring 0 s over $1 \mathrm{~s}$ in the mask, which is caused by the bias towards the larger of $S$ and $R$. The CHF loss function is able to remove this bias and provides a balance between increasing HITs without increasing as many FAs.

Table 4: Classification accuracy (in \%), HIT-FA (in \%) and ESTOI scores for the GRID dataset in babble and factory noise at $-5 \mathrm{~dB}, 0 \mathrm{~dB}$ and $+5 \mathrm{~dB}$.

\begin{tabular}{|c|c|c|c|c|c|}
\hline \multicolumn{2}{|c|}{ Noise (dB) } & Loss & Acc & HIT-FA (FA) & ESTOI \\
\hline \multirow{12}{*}{ babble } & \multirow{4}{*}{-5} & $\mathrm{CE}$ & 89.7 & $66.7 \quad(4.6)$ & 46.9 \\
\hline & & $\mathrm{HF}$ & 84.8 & $68.0(14.5)$ & 42.6 \\
\hline & & CHF & 88.3 & $71.7 \quad(9.5)$ & 46.1 \\
\hline & & \multicolumn{3}{|c|}{ unprocessed audio } & 20.3 \\
\hline & \multirow{4}{*}{0} & $\mathrm{CE}$ & 91.8 & $74.7 \quad(4.1)$ & 62.4 \\
\hline & & $\mathrm{HF}$ & 88.7 & $77.4(11.2)$ & 60.3 \\
\hline & & $\mathrm{CHF}$ & 90.6 & $\mathbf{7 9 . 5} \quad(8.7)$ & 62.8 \\
\hline & & \multicolumn{3}{|c|}{ unprocessed audio } & 33.9 \\
\hline & \multirow{4}{*}{+5} & $\mathrm{CE}$ & 92.6 & $77.6 \quad(4.0)$ & 72.2 \\
\hline & & $\mathrm{HF}$ & 90.1 & $81.0 \quad(10.3)$ & 72.6 \\
\hline & & $\mathrm{CHF}$ & 91.4 & $82.6 \quad(8.5)$ & 74.1 \\
\hline & & \multicolumn{3}{|c|}{ unprocessed audio } & 49.8 \\
\hline \multirow{12}{*}{ factory } & \multirow{4}{*}{-5} & $\mathrm{CE}$ & 92.8 & $69.1 \quad(2.7)$ & 44.8 \\
\hline & & $\mathrm{HF}$ & 89.4 & $74.1 \quad(9.4)$ & 40.9 \\
\hline & & $\mathrm{CHF}$ & 91.1 & $75.7 \quad(7.2)$ & 43.8 \\
\hline & & \multicolumn{3}{|c|}{ unprocessed audio } & 20.1 \\
\hline & \multirow{4}{*}{0} & $\mathrm{CE}$ & 94.4 & $76.9 \quad(2.5)$ & 58.7 \\
\hline & & $\mathrm{HF}$ & 91.3 & $79.9 \quad(8.0)$ & 57.2 \\
\hline & & $\mathrm{CHF}$ & 92.9 & $83.2 \quad(6.4)$ & 60.1 \\
\hline & & \multicolumn{3}{|c|}{ unprocessed audio } & 33.5 \\
\hline & \multirow{4}{*}{+5} & $\mathrm{CE}$ & 95.1 & $80.3 \quad(2.4)$ & 66.9 \\
\hline & & $\mathrm{HF}$ & 92.1 & $83.9 \quad(7.9)$ & 68.1 \\
\hline & & $\mathrm{CHF}$ & 93.6 & $86.6 \quad(6.2)$ & 70.6 \\
\hline & & \multicolumn{3}{|c|}{ unproce } & 49.9 \\
\hline
\end{tabular}

Comparing now the intelligibility as measured by ESTOI, the CE loss function outperforms the HF loss function at lower SNRs while the HF loss function is better at the higher $5 \mathrm{~dB}$ SNR. Even though the HF loss function outperforms CE with regards to the HIT-FA rate across all SNRs, the large number of FAs introduced by the HF loss function reduces the intelligibility to be lower than CE at low SNRs. This shows that even a large increase in HITs does not compensate for a large increase in FAs, which are more detrimental to intelligibility at low SNR than at high SNR. Considering now the performance of the hybrid CHF loss function, this outperforms both CE and $\mathrm{HF}$ at SNRs above $-5 \mathrm{~dB}$ and is slightly worse than $\mathrm{CE}$ at $-5 \mathrm{~dB}$. The CHF loss function had highest HIT-FA rate across all SNR, confirming that increasing the HIT-FA rate does increase intelligibility, but the number of FAs introduced affects the resulting intelligibility. Reducing FAs at low SNRs is critical whereas a higher HIT rate is more important at high SNRs.

Overall, with intelligibility being the main focus, all loss functions provide large gains in intelligibility compared to that of the unprocessed audio. If the SNR is very low, CE is the loss function of choice, however at all other SNRs, CHF is the best performing loss function. CHF also provides a strong balance between both classification accuracy and the HIT-FA rate.

\subsection{Further analysis of perceptual loss functions}

From the experiments in Section 4.2, loss functions CE and $\mathrm{CHF}$ are selected for further analysis in a larger vocabulary dataset. Experiments are performed in babble noise at SNRs of $-5 \mathrm{~dB}, 0 \mathrm{~dB}$ and $+5 \mathrm{~dB}$, with LC set to $5 \mathrm{~dB}$ lower than the select SNR using the RM-3000 dataset. Table 5 shows objective performance across all experiments.

Table 5: Classification accuracy (in \%), HIT-FA (in \%) and ESTOI scores for the RM-3000 dataset in babble noise at $-5 \mathrm{~dB}$, $0 \mathrm{~dB}$ and $+5 \mathrm{~dB}$.

\begin{tabular}{|c|c|c|c|c|c|}
\hline \multicolumn{2}{|c|}{ Noise (dB) } & Loss & Acc & HIT-FA (FA) & ESTOI \\
\hline \multirow{9}{*}{ babble } & \multirow{3}{*}{-5} & $\mathrm{CE}$ & 90.3 & $71.2 \quad(4.8)$ & 46.9 \\
\hline & & $\mathrm{CHF}$ & 88.8 & $\mathbf{7 6 . 2}(10.6)$ & 46.5 \\
\hline & & \multicolumn{3}{|c|}{ unprocessed audio } & 22.0 \\
\hline & \multirow{3}{*}{0} & $\mathrm{CE}$ & 91.7 & $76.2 \quad(4.6)$ & 59.6 \\
\hline & & $\mathrm{CHF}$ & 90.5 & $80.4 \quad(9.2)$ & 60.5 \\
\hline & & \multicolumn{3}{|c|}{ unprocessed audio } & 35.4 \\
\hline & \multirow{3}{*}{+5} & $\mathrm{CE}$ & 92.4 & $78.9 \quad(4.5)$ & 68.8 \\
\hline & & $\mathrm{CHF}$ & 91.3 & $\mathbf{8 2 . 7} \quad(8.8)$ & 70.8 \\
\hline & & \multicolumn{3}{|c|}{ unprocessed audio } & 50.7 \\
\hline
\end{tabular}

Performance of the two loss functions on the RM-3000 dataset are found to follow the same trends as the experiments with GRID, even though this task is more challenging. Applying paired comparisons using Tukey's HSD [35], found the improvement of the hybrid CHF loss function over the CE loss function to be statistically significant $(\mathrm{p}<0.05)$ at both $5 \mathrm{~dB}$ and $0 \mathrm{~dB}$, while at $-5 \mathrm{~dB}$ no statistical difference was found.

\section{Conclusions}

When applied to mask estimation, the conventional cross entropy loss function aims to maximise the accuracy of the mask. In this work, we proposed two new perceptually motivated loss functions that consider the HIT-FA rate as this has been shown to relate more closely to intelligibility than mask accuracy. The first loss function (HF) was based solely on maximising the HIT-FA rate and whilst this was achieved, ESTOI performance was generally lower than the CE loss function due to a larger number of false alarms being introduced. The second loss function (CHF) aimed to combine maximisation of mask accuracy and HIT-FA rate and was able to reduce the bias found within binary cross-entropy by adjusting the ratio between $1 \mathrm{~s}$ and $0 \mathrm{~s}$ inspired by HIT-FA. Evaluation on both small (GRID) and large (RM-3000) vocabulary datasets found that the proposed CHF loss function gave highest HIT-FA and ESTOI in the majority of test conditions, outperforming both CE and HF loss functions.

\section{Acknowledgements}

We wish to thank the UK Home Office - Centre for Applied Science and Technology, for supporting this work. The research presented in this paper was carried out on the High Performance Computing Cluster supported by the Research and Specialist Computing Support service at the University of East Anglia. 


\section{References}

[1] J. Barker, L. Josifovski, M. Cooke, and P. D. Green, "Soft decisions in missing data techniques for robust automatic speech recognition." in INTERSPEECH, 2000, pp. 373-376.

[2] J. Barker, M. Cooke, and P. D. Green, "Robust ASR based on clean speech models: an evaluation of missing data techniques for connected digit recognition in noise." in INTERSPEECH, 2001, pp. 213-217.

[3] B. C. Moore, Cochlear hearing loss: physiological, psychological and technical issues. John Wiley \& Sons, 2007.

[4] P. C. Loizou, Speech enhancement: theory and practice. CRC press, 2013

[5] Y. Hu and P. C. Loizou, "A comparative intelligibility study of single-microphone noise reduction algorithms." The Journal of the Acoustical Society of America, vol. 122, no. 3, pp. 1777-1786, 2007.

[6] H. Levitt, "Noise reduction in hearing aids: a review." Journal of rehabilitation research and development, vol. 38 , no. 1 , p. 111 , 2001.

[7] D. Wang and G. J. Brown, Computational auditory scene analysis: Principles, algorithms, and applications. Wiley-IEEE Press, 2006.

[8] A. S. Bregman, Auditory scene analysis: The perceptual organization of sound. $\quad$ MIT press, 1994.

[9] M. Ahmadi, V. L. Gross, and D. G. Sinex, "Perceptual learning for speech in noise after application of binary time-frequency masks." The Journal of the Acoustical Society of America, vol. 133, no. 3, pp. 1687-1692, 2013.

[10] D. S. Brungart, P. S. Chang, B. D. Simpson, and D. Wang, "Isolating the energetic component of speech-on-speech masking with ideal time-frequency segregation." The Journal of the Acoustical Society of America, vol. 120, no. 6, pp. 4007-4018, 2006.

[11] N. Li and P. C. Loizou, "Factors influencing intelligibility of ideal binary-masked speech: Implications for noise reduction." The Journal of the Acoustical Society of America, vol. 123, no. 3, pp. 1673-1682, 2008 .

[12] D. Wang, U. Kjems, M. S. Pedersen, J. B. Boldt, and T. Lunner, "Speech intelligibility in background noise with ideal binary time-frequency masking." The Journal of the Acoustical Society of America, vol. 125, no. 4, pp. 2336-2347, 2009.

[13] K. Han and D. Wang, "A classification based approach to speech segregation." The Journal of the Acoustical Society of America, vol. 132, no. 5, pp. 3475-3483, 2012.

[14] C. Valentini-Botinhao, Z. Wu, and S. King, "Towards minimum perceptual error training for DNN-based speech synthesis." in $I N$ TERSPEECH, 2015, pp. 869-873.

[15] F. Weninger, J. R. Hershey, J. Le Roux, and B. Schuller, "Discriminatively trained recurrent neural networks for single-channel speech separation," in Signal and Information Processing (GlobalSIP), 2014 IEEE Global Conference on. IEEE, 2014, pp. 577_ 581 .

[16] H. Erdogan, J. R. Hershey, S. Watanabe, and J. Le Roux, "Phasesensitive and recognition-boosted speech separation using deep recurrent neural networks," in Acoustics, Speech and Signal Processing (ICASSP), 2015 IEEE International Conference on. IEEE, 2015, pp. 708-712.

[17] J. Chen, Y. Wang, and D. Wang, "A feature study for classification-based speech separation at low signal-to-noise ratios." Audio, Speech, and Language Processing, IEEE/ACM Transactions on, vol. 22, no. 12, pp. 1993-2002, 2014

[18] E. W. Healy, S. E. Yoho, J. Chen, Y. Wang, and D. Wang, "An algorithm to increase speech intelligibility for hearing-impaired listeners in novel segments of the same noise type." The Journal of the Acoustical Society of America, vol. 138, no. 3, pp. 16601669,2015
[19] G. Kim, Y. Lu, Y. Hu, and P. C. Loizou, "An algorithm that improves speech intelligibility in noise for normal-hearing listeners.' The Journal of the Acoustical Society of America, vol. 126, no. 3 , pp. 1486-1494, 2009.

[20] E. W. Healy, S. E. Yoho, Y. Wang, and D. Wang "An algorithm to improve speech recognition in noise for hearing-impaired listeners." The Journal of the Acoustical Society of America, vol. 134, no. 4, pp. 3029-3038, 2013.

[21] J. Chen, Y. Wang, and D. Wang, "Noise perturbation for supervised speech separation." Speech Communication, vol. 78, pp. 110, 2016.

[22] Y. Wang and D. Wang, "Towards scaling up classification-based speech separation." Audio, Speech, and Language Processing, IEEE Transactions on, vol. 21, no. 7, pp. 1381-1390, 2013.

[23] R. Y. Rubinstein and D. P. Kroese, The cross-entropy method: a unified approach to combinatorial optimization, Monte-Carlo simulation and machine learning. Springer Science \& Business Media, 2013.

[24] R. Patterson, I. Nimmo-Smith, J. Holdsworth, and P. Rice, "An efficient auditory filterbank based on the gammatone function." in a meeting of the IOC Speech Group on Auditory Modelling at RSRE, vol. 2, no. 7, 1987.

[25] B. Kollmeier and R. Koch, "Speech enhancement based on physiological and psychoacoustical models of modulation perception and binaural interaction." The Journal of the Acoustical Society of America, vol. 95, no. 3, pp. 1593-1602, 1994.

[26] J. Tchorz and B. Kollmeier, "SNR estimation based on amplitude modulation analysis with applications to noise suppression." Speech and Audio Processing, IEEE Transactions on, vol. 11, no. 3, pp. 184-192, 2003.

[27] H. Hermansky and N. Morgan, "RASTA processing of speech." Speech and Audio Processing, IEEE Transactions on, vol. 2, no. 4, pp. 578-589, 1994.

[28] ETSI, "Speech Processing, Transmission and Quality Aspects (STQ); Distributed speech recognition; Advanced front-end feature extraction algorithm; Compression algorithms." ETSI STQAurora DSR Working Group, ES 202050 version 1.1.1, Oct. 2002

[29] S. Furui, "Speaker-independent isolated word recognition using dynamic features of speech spectrum," IEEE Transactions on Acoustics, Speech, and Signal Processing., vol. 34, no. 1, pp. $52-$ 59, Feb 1986.

[30] B. Hanson and T. Applebaum, "Robust speaker-independent word recognition using static, dynamic and acceleration features: Experiments with Lombard and noisy speech." in Acoustics, Speech, and Signal Processing, 1990. ICASSP-90., 1990 International Conference on. IEEE, 1990, pp. 857-860.

[31] M. Cooke, J. Barker, S. Cunningham, and X. Shao, "An audiovisual corpus for speech perception and automatic speech recognition." The Journal of the Acoustical Society of America, vol. 120 , no. 5, pp. 2421-2424, 2006

[32] D. Howell, S. Cox, and B. Theobald, "Visual units and confusion modelling for automatic lip-reading," Image and Vision Computing, vol. 51, pp. 1-12, 2016.

[33] P. Price, W. M. Fisher, J. Bernstein, and D. S. Pallett, "The darpa 1000-word resource management database for continuous speech recognition," in Acoustics, Speech, and Signal Processing, 1988. ICASSP-88., 1988 International Conference on. IEEE, 1988, pp. 651-654.

[34] J. Jensen and C. H. Taal, "An algorithm for predicting the intelligibility of speech masked by modulated noise maskers,' IEEE/ACM Transactions on Audio, Speech, and Language Processing, vol. 24, no. 11, pp. 2009-2022, 2016.

[35] J. W. Tukey, "Comparing individual means in the analysis of variance," Biometrics, pp. 99-114, 1949. 